\title{
Organisation und Verlauf
}

Der Bundesweite Überwachungsplan (BÜp) ist ein für ein Jahr festgelegter Plan über die zwischen den Ländern abgestimmte Durchführung von amtlichen Kontrollen zur Überprüfung der Einhaltung der lebensmittelrechtlichen, weinrechtlichen und tabakrechtlichen Vorschriften. Er kann Programme zu Produkt- und Betriebskontrollen oder eine Kombination aus beidem enthalten. Im Gegensatz zum Monitoring nach §§ 50-52 des Lebensmittelund Futtermittelgesetzbuchs (LFGB) ist der BÜp ein risikoorientiertes Überwachungsprogramm. Das heißt, dass die Auswahl der zu untersuchenden Proben und der zu kontrollierenden Betriebe gezielt auf Basis einer Risikoanalyse erfolgt. Im Rahmen des BÜp können Lebensmittel, Kosmetika, Bedarfsgegenstände und Tabakerzeugnisse untersucht werden. Die Untersuchungen können dabei beispielsweise die folgenden Aspekte abdecken: chemische Parameter, mikrobiologische Parameter, die Anwendung bestimmter Herstellungsverfahren oder die Überprüfung von Kennzeichnungselementen. Betriebskontrollen werden vorrangig zur Prüfung der Einhaltung hygienerechtlicher Vorgaben, der Rückverfolgbarkeit, der Zusammensetzung und der Kennzeichnung der Produkte durchgeführt.

Ziel des BÜp ist es, bundesweite Aussagen über die Einhaltung lebensmittelrechtlicher, weinrechtlicher und tabakrechtlicher Vorschriften einschließlich des Täuschungsschutzes zu erhalten. Gerade bei neuen gesetzlichen Regelungen wie beispielsweise neu eingeführten Höchstgehalten oder geänderten Kennzeichnungsvorschriften sind bundesweite Aussagen zum Grad der Umsetzung bzw. der Verstöße von Interesse. Außerdem werden die im BÜp erhobenen Daten regelmäßig zur Klärung von aktuellen Fragestellungen verwendet. So kann z. B. untersucht werden, ob und in welchem Ausmaß inakzeptable Kontaminationen in Produkten vorliegen, was ggf. zur Festlegung vorläufiger Höchstgehalte führen kann.

Die Länder, das Bundesministerium für Ernährung und Landwirtschaft (BMEL), das Bundesinstitut für Risikobewertung (BfR) sowie das Bundesamt für Verbrau- cherschutz und Lebensmittelsicherheit (BVL) können Vorschläge für BÜp-Programme einreichen. Die Entscheidung, welche dieser Programme tatsächlich durchgeführt werden sollen, wird von einer Expertengruppe getroffen, in der die oben genannten Institutionen vertreten sind.

Da aufgrund regionaler Unterschiede nicht alle Fragestellungen für alle Länder gleich relevant sind, entscheiden diese eigenständig, an welchen BÜp-Programmen sie sich mit wie vielen Proben beteiligen. Eine Umsetzung der Programme erfolgt nur dann, wenn mindestens zwei Länder eine Beteiligung daran zusagen. Auf der Basis der ausgewählten Programme wird vom BVL der BÜp erstellt.

Die im Rahmen des BÜp erhobenen Daten werden dem BVL übermittelt. Nach Überprüfung der Vollständigkeit der von den Ländern übermittelten Daten werden die Einzeldaten zu den einzelnen Programmen zusammengestellt. Nach einer ersten Plausibilitätsprüfung im BVL werden die zusammengestellten Einzeldaten den Programminitiatoren übermittelt, die ihrerseits eine weitere Plausibilitätsprüfung der Daten vornehmen. Gleichzeitig mit den Einzeldaten erhalten die Programminitiatoren einen Vorschlag für die tabellarische Darstellung der Auswertungen. Entsprechend der Rückmeldung des jeweiligen Programminitiators werden die Auswertungen der Daten in der Regel im BVL vorgenommen. Anhand der vom BVL übermittelten Auswertungen erstellen die Programminitiatoren einen Berichtsentwurf. Die dem BVL übermittelten Berichtsentwürfe werden mit den allgemeinen Kapiteln zu einem Gesamtberichtsentwurf zusammengeführt und der BÜp-Redaktionsgruppe übermittelt. Die in der Redaktionsgruppe abgestimmte Fassung wird anschließend den obersten Landesbehörden zur Zustimmung übermittelt. Nach der gemeinsamen öffentlichen Vorstellung des Endberichtes durch das BVL und den LAV-Vorsitz steht dieser gemeinsame Bericht des Bundes und der Länder sowohl in gedruckter Form als auch elektronisch unter www.bvl.bund.de/buep allen Interessierten zur Verfügung. 